\title{
SCALAR CURVATURE OF MINIMAL HYPERSURFACES IN A SPHERE
}

\author{
Si-Ming Wei And Hong-Wei Xu
}

\begin{abstract}
We first extend the well-known scalar curvature pinching theorem due to Peng-Terng, and prove that if $M$ a closed minimal hypersurface in $S^{n+1}(n=6,7)$, then there exists a positive constant $\delta(n)$ depending only on $n$ such that if $n \leq S \leq n+\delta(n)$, then $S \equiv n$, i.e., $M$ is one of the Clifford torus $S^{k}\left(\sqrt{\frac{k}{n}}\right) \times S^{n-k}\left(\sqrt{\frac{n-k}{n}}\right), k=1,2, \ldots, n-1$. Secondly, we point out a mistake in Ogiue and Sun's paper in which they claimed that they had solved the open problem proposed by Peng and Terng.
\end{abstract}

\section{Introduction}

Let $M$ be an $n$-dimensional closed minimal hypersurface in an $(n+1)$-dimensional unit sphere $S^{n+1}$. Denote by $S$ the squared length of the second fundamental form of $M$ and $R$ its scalar curvature. So $R=n(n-1)-S$. The famous rigidity theorem due to Simons, Lawson, Chern, do Carmo and Kobayashi $[4,5,10]$ says that if $S \leq n$, then $S \equiv 0$, or $S \equiv n$. i.e., $\mathrm{M}$ is the great sphere $S^{n}$, or the Clifford torus $S^{k}\left(\sqrt{\frac{k}{n}}\right) \times$ $S^{n-k}\left(\sqrt{\frac{n-k}{n}}\right)$. Further discussions in this direction have been carried out by many other authors $[1,3,6,11,12,13,14]$, etc.. On the other hand, many geometers have been interested in the question whether there are several scalar curvature pinching phenomena for closed minimal hypersurfaces in a unit sphere. In [8], Peng and Terng proved that if the scalar curvature of $M$ is a constant, then there exists a positive constant $\alpha(n)$ depending only on $n$ such that if $n \leq S \leq n+\alpha(n)$, then $S=n$. Later Cheng and Yang [2] improved the pinching constant $\alpha(n)$ to $n / 3$. More general, Peng and Terng [9] obtained an important pinching theorem for minimal hypersurfaces without assumption that the scalar curvature is a constant. Precisely, they proved that if $M^{n}(n \leq 5)$ is a closed minimal hypersurface in $S^{n+1}$, then there exists a positive constant $\delta(n)$ depending only on $n$ such that if $n \leq S \leq n+\delta(n)$, then $S \equiv n$. The following problem proposed by Peng and Terng [9] is very attractive.

Open Problem. Let $M$ be an n-dimensional closed minimal hypersurface in $S^{n+1}, n \geq 6$. Does there exist a positive constant $\delta(n)$ depending only on $n$ such that if $n \leq S \leq n+\delta(n)$, then $S \equiv n$, i.e., $M$ is one of the Clifford torus $S^{k}\left(\sqrt{\frac{k}{n}}\right) \times$ $S^{n-k}\left(\sqrt{\frac{n-k}{n}}\right), k=1,2, \ldots, n-1$ ?

Received by the editors December 5, 2005.

Research supported by the NNSF of China, Grant No. 10231010; the Trans-Century Training Programme Foundation for Talents by the Ministry of Education of China; and Natural Science Foundation of Zhejiang Province, Grant No. 101037.

Key words: Minimal hypersurfaces, Scalar curvature, Second fundamental forms, Clifford torus. 
In this note, we solve the open problem for $n=6,7$, and prove the following pinching theorem for minimal hypersurfaces in unit spheres of dimensions 7 and 8 .

Theorem. Let $M$ be an $n$-dimensional closed minimal hypersurface in $S^{n+1}, n=$ 6,7. Then there exists a positive constant $\delta(n)$ depending only on $n$ such that if $n \leq S \leq n+\delta(n)$, then $S \equiv n$, i.e., $M$ is one of the Clifford torus $S^{k}\left(\sqrt{\frac{k}{n}}\right) \times$ $S^{n-k}\left(\sqrt{\frac{n-k}{n}}\right), k=1,2, \ldots, n-1$. Here $\delta(6)=\frac{1}{76}$ and $\delta(7)=\frac{1}{1126}$.

Our theorem generalize the scalar curvature pinching theorem due to Peng and Terng [9] from the case $n \leq 5$ to $n \leq 7$. Up to now, the open problem for $n \geq 8$ is still open.

In [7], Ogiue and Sun claimed that they had solved the open problem for arbitrary $n$. Unfortunately, there is a fatal mistake in their proof. In section 4 , we point out their mistake.

\section{Fundamental formulas for minimal hypersurfaces in a sphere}

Throughout this paper let $M$ be an $n$-dimensional closed minimal hypersurface in an $(n+1)$-dimensional unit sphere $S^{n+1}$. We shall make use of the following convention on the range of indices:

$$
1 \leq A, B, C, \cdots \leq n+1, \quad 1 \leq i, j, k, \cdots \leq n .
$$

Choose a local orthonormal frame field $\left\{e_{A}\right\}$ in $\mathrm{S}^{n+1}$ such that, restricted to $M$, the $e_{i}$ s are tangent to $M$. Let $\left\{\omega_{A}\right\}$ be the dual frame fields of $\left\{e_{A}\right\}$ and $\left\{\omega_{A B}\right\}$ the connection 1-forms of $\mathrm{S}^{n+1}$ respectively. Restricting these forms to $M$, we have $\omega_{n+1 i}=\sum_{j} h_{i j} \omega_{j}, h_{i j}=h_{j i}$. Let $R$ and $h$ be the scalar curvature and the second fundamental form of $M$ respectively. Denote by $S$ the squared length of $h$ and $H$ the mean curvature of $M$. Then we have

$$
\begin{gathered}
h=\sum_{i, j} h_{i j} \omega_{i} \otimes \omega_{j}, \quad S=\sum_{i, j} h_{i j}^{2} . \\
H=\frac{1}{n} \sum_{i} h_{i i}=0, \quad R=n(n-1)-S .
\end{gathered}
$$

Denote by $h_{i j k}, h_{i j k l}$ and $h_{i j k l m}$ the first, second and third covariant derivatives of the second fundamental form tensor $h_{i j}$. Then

$$
\begin{gathered}
\nabla h=\sum_{i, j, k} h_{i j k} \omega_{i} \otimes \omega_{j} \otimes \omega_{k}, \quad h_{i j k}=h_{i k j} . \\
h_{i j k l}=h_{i j l k}+\sum_{m} h_{m j} R_{m i k l}+\sum_{m} h_{i m} R_{m j k l} . \\
h_{i j k l m}=h_{i j k m l}+\sum_{r} h_{r j k} R_{r i l m}+\sum_{r} h_{i r k} R_{r j l m}+\sum_{r} h_{i j r} R_{r k l m} .
\end{gathered}
$$

For an arbitrary fixed point $x \in M$, we take orthonormal frames such that $h_{i j}=\lambda_{i} \delta_{i j}$ for all $i, j$. Then

$$
\sum_{i} \lambda_{i}=0, \quad \sum_{i} \lambda_{i}^{2}=S
$$


Following $[4,9]$, we have

$$
\begin{gathered}
\frac{1}{2} \triangle S=|\nabla h|^{2}+S(n-S) . \\
\frac{1}{2} \triangle\left(|\nabla h|^{2}\right)=\left|\nabla^{2} h\right|^{2}+(2 n+3-S)|\nabla h|^{2}+3(2 B-A)-\frac{3}{2}|\nabla S|^{2} . \\
\lambda_{k}^{2}-4 \lambda_{i} \lambda_{k}<\frac{\sqrt{17}+1}{2} S, \quad 1 \leq i, k \leq n . \\
3(A-2 B) \leq \frac{\sqrt{17}+1}{2} S|\nabla h|^{2}, \\
\int_{M}\left[\left(S-2 n-\frac{3}{2}\right)|\nabla h|^{2}+\frac{3}{2}(A-2 B)+\frac{9}{8}|\nabla S|^{2}\right] d M \geq 0,
\end{gathered}
$$

where $A=\sum_{i, j, k} h_{i j k}^{2} \lambda_{i}^{2}$ and $B=\sum_{i, j, k} h_{i j k}^{2} \lambda_{i} \lambda_{j}$.

\section{Proof of Theorem}

The crucial point in our proof is to give a sharper pointwise estimate of $3(A-2 B)$ in terms of $S$ and $|\nabla h|^{2}$ by using new method. The following lemmas will be used in the proof of the theorem.

Lemma 3.1. Let $M$ be an n-dimensional closed minimal hypersurface in the unit sphere $S^{n+1}, n \geq 6$. Suppose that

$$
3(A-2 B) \leq t(n) S|\nabla h|^{2},
$$

where $t(n)$ is a number depending only on $n$ satisfying $0 \leq t(n)<2+\frac{3}{n}$. Then there exists a positive constant $\delta(n)$ such that if $n \leq S \leq n+\delta(n)$, then $S \equiv n$.

Proof. By the assumption, we have

$$
\frac{6-[2 t(n)-4] n}{9}>0 .
$$

We choose a positive constant $\delta(n)$ depending only on $n$ satisfying

$$
0<\delta(n)<\frac{6-[2 t(n)-4] n}{9} .
$$

It follows from the assumption $n \leq S(x) \leq n+\delta$ that

$$
\int_{M}|\nabla S|^{2} d M=2 \int_{M}\left[S^{2}(S-n)-S|\nabla h|^{2}\right] d M \leq 2 \int_{M}(n+\delta-S)|\nabla h|^{2} d M .
$$

From (2.11), (3.1) and (3.3), we obtain

$$
0 \leq \int_{M}\left(\frac{2 t(n)-5}{4} S-\frac{6-n-9 \delta(n)}{4}\right)|\nabla h|^{2} d M .
$$

Since

$$
t(n)<2+\frac{3}{n} \leq \frac{5}{2}
$$


we get

$$
\begin{aligned}
0 & \leq \int_{M}[(2 t(n)-5) S-(6-n-9 \delta(n)])|\nabla h|^{2} d M \\
& \leq \int_{M}[(2 t(n)-4) n+(9 \delta(n)-6)]|\nabla h|^{2} d M,
\end{aligned}
$$

which implies

$$
\int_{M}|\nabla h|^{2} d M \leq 0
$$

Hence $|\nabla h|^{2}=0$. It's easy to see from (2.7) that $S \equiv n$.

Remark 1. Under the assumption of Lemma 3.1, if $t(n)=2$, then the pinching constant $\delta(n)=\frac{2}{3}$, which is a universal positive constant independent of $n$.

Lemma 3.2. Let $M$ be a closed minimal hypersurface in a 7-dimensional unit sphere $S^{7}$. Then

$$
\sum_{i} h_{i i k}^{2} \Phi(i, k) \leq 2.49 S \cdot \sum_{i} h_{i i k}^{2}, 1 \leq k \leq 6,
$$

where

$$
\Phi(i, k)= \begin{cases}\lambda_{k}^{2}-4 \lambda_{i} \lambda_{k}, & i \neq k \\ 2 S, & i=k\end{cases}
$$

Proof. Without loss of generality, we suppose that $k=1$. If $\Phi(i, 1) \leq 2.49 S$ for any $i$, or $\sum_{i} h_{i i 1}^{2}=0$, it is easy to get (3.7). Otherwise, without loss of generality, we suppose that $\Phi(2,1)>2.49 S$. Then

$$
\begin{aligned}
\frac{\sqrt{17}+1}{2} S & \geq \frac{\sqrt{17}+1}{2}\left(\lambda_{1}^{2}+\lambda_{2}^{2}\right) \\
& \geq \lambda_{1}^{2}-2\left(\sqrt{\frac{\sqrt{17}-1}{2}} \lambda_{1}\right)\left(\sqrt{\frac{\sqrt{17}+1}{2} \lambda_{2}}\right) \\
& =\Phi(2,1)>2.49 S .
\end{aligned}
$$

This implies

$$
\lambda_{m}^{2} \leq S-\left(\lambda_{1}^{2}+\lambda_{2}^{2}\right)<S-\frac{2.49}{2.57} S=\frac{8}{257} S, m=3,4,5,6 .
$$

By (3.9) we have

$$
\Phi(m, 1)=\lambda_{1}^{2}-4 \lambda_{1} \lambda_{m}<S+4 \cdot \sqrt{\frac{8}{257} S} \cdot \sqrt{S}<2 S, m=3,4,5,6 .
$$

Since $M$ is a minimal hypersurface, we have $\sum_{i} h_{i i}=0$. Hence

$$
h_{221}=-\sum_{i \neq 2} h_{i i 1},
$$

which implies

$$
\sum_{i \neq 2} h_{i i 1}^{2} \geq \frac{h_{221}^{2}}{5} .
$$


It follows from (3.8), (3.10) and (3.11) that

$$
\begin{aligned}
2.49 S \sum_{i} h_{i i 1}^{2} & \geq 2.49 S h_{221}^{2}+0.49 S \cdot \frac{h_{221}^{2}}{5}+\sum_{i \neq 2} h_{i i 1}^{2} \Phi(i, 1) \\
& \geq \sum_{i} h_{i i 1}^{2} \Phi(i, 1) .
\end{aligned}
$$

Lemma 3.3. Let $M$ be a closed minimal hypersurface in an 8-dimensional unit sphere $S^{8}$. Then

$$
\sum_{i} h_{i i k}^{2} \Phi(i, k) \leq 2.428 S \cdot \sum_{i} h_{i i k}^{2}, 1 \leq k \leq 7
$$

where

$$
\Phi(i, k)= \begin{cases}\lambda_{k}^{2}-4 \lambda_{i} \lambda_{k}, & i \neq k \\ 1.62 S, & i=k\end{cases}
$$

Proof. Without loss of generality, we suppose that $k=1$. If $\Phi(i, 1) \leq 2.428 S$ for any $i$, or $\sum_{i} h_{i i 1}^{2}=0$, it is easy to get (3.13). Otherwise, without loss of generality, we suppose that $\Phi(2,1)>2.428 S$. Then

$$
\begin{aligned}
\frac{\sqrt{17}+1}{2} S & \geq \frac{\sqrt{17}+1}{2}\left(\lambda_{1}^{2}+\lambda_{2}^{2}\right) \\
& \geq \lambda_{1}^{2}-4 \lambda_{1} \lambda_{2}>2.428 S .
\end{aligned}
$$

It follows from the above that

$$
\lambda_{m}^{2} \leq S-\left(\lambda_{1}^{2}+\lambda_{2}^{2}\right)<S-\frac{2.428}{2.562} S=\frac{67}{1281} S,
$$

where $3 \leq m \leq 7$. On the other hand, we have

$$
\lambda_{1}^{2}+\left(\lambda_{1}^{2}+4 \lambda_{2}^{2}\right) \geq \lambda_{1}^{2}-4 \lambda_{1} \lambda_{2}>2.428 S .
$$

This implies

$$
\lambda_{1}^{2} \leq S-\lambda_{2}^{2}<S-\frac{2.428 S-2\left(\lambda_{1}^{2}+\lambda_{2}^{2}\right)}{2} \leq 0.786 S .
$$

From (3.15) and (3.16) we have

$$
\Phi(m, 1)=\lambda_{1}^{2}-4 \lambda_{1} \lambda_{m}<0.786 S+4 \cdot \sqrt{\frac{67}{1281} S} \cdot \sqrt{0.786 S} \leq 1.62 S,
$$

where $3 \leq m \leq 7$. Since $M$ is a minimal hypersurface, we have $\sum_{i} h_{i i}=0$, which implies

$$
\sum_{i \neq 2} h_{i i 1}^{2} \geq \frac{h_{221}^{2}}{6} .
$$


From (3.14), (3.17) and (3.18) we obtain

$$
\begin{aligned}
2.428 S \sum_{i} h_{i i 1}^{2} & \geq 2.428 S h_{221}^{2}+0.808 S \cdot \frac{h_{221}^{2}}{6}+\sum_{i \neq 2} h_{i i 1}^{2} \Phi(i, 1) \\
& \geq \sum_{i} h_{i i 1}^{2} \Phi(i, 1) .
\end{aligned}
$$

Now we are in a position to give the proof of our theorem.

Proof of Theorem. (i) When $n=6$, it follows from Lemma 3.2 that

$$
\begin{aligned}
& 3 \sum_{i \neq k} h_{i i k}^{2}\left(\lambda_{k}^{2}-4 \lambda_{k} \lambda_{i}\right)+\sum_{i} h_{i i i}^{2}\left(-3 \lambda_{i}^{2}\right) \\
& =3 \sum_{k}\left(\sum_{i} h_{i i k}^{2} \phi(i, k)\right)-3 \sum_{k} h_{k k k}^{2} \cdot 2 S+\sum_{i} h_{i i i}^{2}\left(-3 \lambda_{i}^{2}\right) \\
& \leq 2.49 S \cdot \sum_{i, k} 3 h_{i i k}^{2}-2.49 S \sum_{k} 2 h_{k k k}^{2} \\
& =2.49 S\left(3 \sum_{i \neq k} h_{i i k}^{2}+\sum_{i} h_{i i i}^{2}\right) .
\end{aligned}
$$

This together with (2.11) implies

$$
\begin{aligned}
3(A-2 B) & =\sum_{\substack{i, j, k \\
\text { dinstinct }}} h_{i j k}^{2}\left[2\left(\lambda_{i}^{2}+\lambda_{j}^{2}+\lambda_{k}^{2}\right)-\left(\lambda_{i}+\lambda_{j}+\lambda_{k}\right)^{2}\right] \\
& +3 \sum_{i \neq k} h_{i i k}^{2}\left(\lambda_{k}^{2}-4 \lambda_{k} \lambda_{i}\right)+\sum_{i} h_{i i i}^{2}\left(-3 \lambda_{i}^{2}\right) \\
& \leq 2 S \sum_{\substack{i, j, k \\
\text { dinstinct }}} h_{i j k}^{2}+2.49 S\left(3 \sum_{i \neq k} h_{i i k}^{2}+\sum_{i} h_{i i i}^{2}\right) \\
& \leq 2.49 S|\nabla h|^{2} .
\end{aligned}
$$

Notice that $\delta(6)=\frac{1}{76}$ and $t(6)=2.49$, we conclude from Lemma 3.1 and (3.21) that $S \equiv 6$, i.e., $M$ is one of the Clifford torus $S^{k}\left(\sqrt{\frac{k}{6}}\right) \times S^{6-k}\left(\sqrt{\frac{6-k}{6}}\right), k=1,2, \ldots, 5$.

(ii) When $n=7$, it follows from Lemma 3.3 that

$$
\begin{aligned}
& 3 \sum_{i \neq k} h_{i i k}^{2}\left(\lambda_{k}^{2}-4 \lambda_{k} \lambda_{i}\right)+\sum_{i} h_{i i i}^{2}\left(-3 \lambda_{i}^{2}\right) \\
& =3 \sum_{k}\left(\sum_{i} h_{i i k}^{2} \phi(i, k)\right)-3 \sum_{k} h_{k k k}^{2} \cdot 1.62 S+\sum_{i} h_{i i i}^{2}\left(-3 \lambda_{i}^{2}\right) \\
& \leq 2.428 \cdot \sum_{i, k} 3 h_{i i k}^{2}-2.428 S \sum_{k} 2 h_{k k k}^{2} \\
& =2.428\left(3 \sum_{i \neq k} h_{i i k}^{2}+\sum_{i} h_{i i i}^{2}\right) .
\end{aligned}
$$


This together with (2.11) implies

$$
\begin{aligned}
3(A-2 B) & =\sum_{\substack{i, j, k \\
\text { dinstinct }}} h_{i j k}^{2}\left[2\left(\lambda_{i}^{2}+\lambda_{j}^{2}+\lambda_{k}^{2}\right)-\left(\lambda_{i}+\lambda_{j}+\lambda_{k}\right)^{2}\right] \\
& +3 \sum_{i \neq k} h_{i i k}^{2}\left(\lambda_{k}^{2}-4 \lambda_{k} \lambda_{i}\right)+\sum_{i} h_{i i i}^{2}\left(-3 \lambda_{i}^{2}\right) \\
& \leq 2 S \sum_{\substack{i, j, k \\
\text { dinstinct }}} h_{i j k}^{2}+2.428 S\left(3 \sum_{i \neq k} h_{i i k}^{2}+\sum_{i} h_{i i i}^{2}\right) \\
& \leq 2.428 S|\nabla h|^{2} .
\end{aligned}
$$

Notice that $\delta(7)=\frac{1}{1126}$ and $t(7)=2.428$, we conclude from Lemma 3.1 and (3.23) that $S \equiv 7$, i.e., $M$ is a Clifford torus. This completes the proof of the theorem.

\section{Notes on Ogiue and Sun's proof}

In [7], Ogiue and Sun claimed that they improved Peng and Terng's pinching theorem for $n(\leq 5)$-dimensional minimal hypersurfaces [9] to the case of arbitrary $n$ :

Let $M$ be an $n$-dimensional closed minimally immersed hypersurface in $S^{n+1}$. Then there exists a constant $\varepsilon(n)=2 n^{2}(n+4) /\left[3(n+2)^{2}\right]$ such that if $n \leq S \leq n+\varepsilon(n)$, then $S \equiv n$ so that $M$ is a Clifford torus.

If the claim were true, definitely it would have been an important contribution to the theory of minimal submanifolds. Unfortunately, there is a fatal mistake in the proof of the key lemma in [7]. Put $g_{3}=\sum_{i, j, k} h_{i j} h_{j k} h_{k i}, g_{4}=\sum_{i, j, k, l} h_{i j} h_{j k} h_{k l} h_{l i}$. This lemma and the sketch of its proof is cited as follows.

Lemma([7]). Let $M$ be an $n$-dimensional closed minimally immersed hypersurface in $S^{n+1}$. If $S \geq n$, then we have

$$
\sum_{i, j, k, l} h_{i j k l}^{2} \geq \frac{3(n+2)}{n(n+4)} S(S-n)^{2}-\frac{3}{n} S^{2}(S-n)+3\left(S g_{4}-g_{3}^{2}-S^{2}\right) .
$$

Proof. Since $M$ is minimal, we have $\sum_{i} h_{i i}=0$ and $\sum_{i, j} h_{i j i j} h_{j j}=0$.

From(1.1)[7] we get $\Delta h_{i i}=(n-S) h_{i i}$ and $\sum_{i, j} h_{i i j j} h_{i i}=S(n-S)$.

Let $f_{i j}=h_{i j i j}$. We consider $f=\sum_{i} f_{i i}^{2}+3 \sum_{i \neq j} f_{i j}^{2}+6 \sum_{i, j}\left(h_{j j}^{2} h_{i i}-h_{i i}^{2} h_{j j}\right) f_{i j}$ as a function of $f_{i j}$. Solve the following problem for the conditional extremum:

$F=\sum_{i} f_{i i}^{2}+3 \sum_{i \neq j} f_{i j}^{2}+6 \sum_{i, j}\left(h_{j j}^{2} h_{i i}-h_{i i}^{2} h_{j j}\right) f_{i j}+\lambda\left[\sum_{i, j} f_{i j} h_{i i}-S(n-S)\right]+\mu \sum_{i, j} f_{i j} h_{i j}$,

where $\lambda$ and $\mu$ are the Lagrange multipliers. It is clear that the critical point of $F$ is the minimum point of $f$. Taking derivatives of $F$ with respect to $f_{i j}$, we get

$$
\begin{gathered}
F_{f_{i j}}=2 f_{i i}+\lambda h_{i i}+\mu h_{i i}=0, \quad i=j, \\
F_{f_{i j}}=6 f_{i j}+6\left(h_{j j}^{2} h_{i i}-h_{i i}^{2} h_{j j}\right)+\lambda h_{i i}+\mu h_{j j}=0, \quad i \neq j,
\end{gathered}
$$


and they satisfy

$$
\sum_{i, j} h_{j j} f_{i j}=0, \sum_{i, j} h_{i i} f_{i j}=S(n-S), \sum_{i} h_{i i}^{2}=S, \sum_{i} h_{i i}=0 .
$$

and so, in view of (2.4)[7]

$$
\begin{gathered}
\sum_{i} f_{i i}^{2}+3 \sum_{i \neq j} f_{i j}^{2}+6 \sum_{i \neq j}\left(h_{j j}^{2} h_{i i}-h_{i i}^{2} h_{j j}\right) f_{i j} \\
=3 \sum_{i \neq j}\left(h_{j j}^{2} h_{i i}-h_{i i}^{2} h_{j j}\right) f_{i j}-\frac{1}{2} \lambda \sum_{i, j} h_{i i} f_{i j} \\
=3 \sum_{i \neq j}\left(h_{j j}^{2} h_{i i}-h_{i i}^{2} h_{j j}\right) f_{i j}+\frac{\lambda}{2} S(S-n) . \\
\ldots \ldots \ldots \cdots \cdots \cdots \cdots \cdots \\
\lambda=\frac{6(n+2)}{n(n+4)}(S-n)-\frac{6}{n} S .
\end{gathered}
$$

(2.10)[7] and (2.15)[7] show that

$$
\begin{aligned}
& \sum_{i} f_{i i}^{2}+3 \sum_{i \neq j} f_{i j}^{2}+6 \sum_{i \neq j}\left(h_{j j}^{2} h_{i i}-h_{i i}^{2} h_{j j}\right) f_{i j} \\
& =\frac{3(n+2)}{n(n+4)} S(S-n)^{2}-\frac{3}{n} S^{2}(S-n)+3 \sum_{i \neq j}\left(h_{j j}^{2} h_{i i}-h_{i i}^{2} h_{j j}\right) f_{i j},
\end{aligned}
$$

and so,

$$
\begin{aligned}
\sum_{i} h_{i i i i}^{2} & +3 \sum_{i \neq j} h_{i j i j}^{2} \geq \frac{3(n+2)}{n(n+4)} S(S-n)^{2}-\frac{3}{n} S^{2}(S-n)-3 \sum_{i \neq j}\left(h_{j j}^{2} h_{i i}-h_{i i}^{2} h_{j j}\right) f_{i j} \\
& =\frac{3(n+2)}{n(n+4)} S(S-n)^{2}-\frac{3}{n} S^{2}(S-n)-3\left(S g_{4}-g_{3}^{2}-S^{2}\right) .
\end{aligned}
$$

Combining (1.4)[7] and (2.16)[7], we get the Lemma.

We see from the above sketch that the key lemma in [7] is derived by computing the minimal value of the function

$$
f=\sum_{i} f_{i i}^{2}+3 \sum_{i \neq j} f_{i j}^{2}+6 \sum_{i, j}\left(h_{j j}^{2} h_{i i}-h_{i i}^{2} h_{j j}\right) f_{i j}
$$

in the domain

$$
\left\{\left(f_{11}, f_{12}, \ldots f_{1 n}, f_{21}, \ldots f_{n n}\right) \mid \sum_{i, j} h_{i j} f_{i j}=0, \sum_{i, j} h_{i i} f_{i j}=S(n-S)\right\} .
$$

Let $P_{0}=\left(\left(f_{11}\right)_{0},\left(f_{12}\right)_{0}, \ldots\left(f_{1 n}\right)_{0},\left(f_{21}\right)_{0}, \ldots\left(f_{n n}\right)_{0}\right)$ be the point where $f$ attains it's minimal value. We see that the exact meaning of the equation above (2.16)[7] is:

$$
\left.f\right|_{p_{0}}=C(n, S)+3 \sum_{i \neq j}\left(h_{j j}^{2} h_{i i}-h_{i i}^{2} h_{j j}\right)\left(f_{i j}\right)_{0},
$$


where

$$
C(n, S)=\frac{3(n+2)}{n(n+4)} S(S-n)^{2}-\frac{3}{n} S^{2}(S-n) .
$$

This implies

$$
\begin{aligned}
f & =\sum_{i} f_{i i}^{2}+3 \sum_{i \neq j} f_{i j}^{2}+6 \sum_{i, j}\left(h_{j j}^{2} h_{i i}-h_{i i}^{2} h_{j j}\right) f_{i j} \\
& \geq C(n, S)+3 \sum_{i \neq j}\left(h_{j j}^{2} h_{i i}-h_{i i}^{2} h_{j j}\right)\left(f_{i j}\right)_{0} .
\end{aligned}
$$

We notice that $f_{i j}$ on the left hand side is different from $\left(f_{i j}\right)_{0}$ on the right hand side. Unfortunately, (2.16)[7] is derived from (4.2) under the additional assumption that $f_{i j}=\left(f_{i j}\right)_{0}$. This is a fatal mistake. In fact, the key lemma [7] is derived from the following assertion.

For $f_{11}, f_{12}, \ldots f_{1 n}, f_{21}, \ldots f_{n n}$ and $h_{11}, h_{22}, \ldots h_{n n}$ satisfying the conditions

$$
\sum_{i, j} h_{j j} f_{i j}=0, \sum_{i, j} h_{i i} f_{i j}=S(n-S), \sum_{i} h_{i i}^{2}=S, \sum_{i} h_{i i}=0,
$$

we always have

$$
\sum_{i} f_{i i}^{2}+3 \sum_{i \neq j} f_{i j}^{2} \geq C(n, S)+3\left(S g_{4}-g_{3}^{2}-S^{2}\right)
$$

Unfortunately, we have the following counter example for the assertion above.

Example 4.1. Set

$$
\begin{gathered}
h_{11}=-h_{22}=-\sqrt{\frac{S}{2}} ; \quad h_{i i}=0, i \geq 3 . \\
f_{i j}=\frac{1}{2}\left(h_{i i}-h_{j j}\right)\left(1+h_{i i} h_{j j}\right)-\frac{S-n}{2(n+4)}\left(h_{i i}+h_{j j}\right), i \neq j . \\
f_{i i}=\frac{3}{n+4}(S-n) h_{i i} .
\end{gathered}
$$

It is easy to see that $f_{i j}$ and $h_{i i}$ satisfy (4.3). On the other hand, we have

$$
\sum_{i} f_{i i}^{2}+3 \sum_{i \neq j} f_{i j}^{2}=C(n, S)+\frac{3}{2}\left(S g_{4}-g_{3}^{2}-\frac{S^{3}}{n}\right)<C(n, S)+3\left(S g_{4}-g_{3}^{2}-S^{2}\right) .
$$

This contradicts with (4.4).

\section{Acknowledgements}

The authors would like to thank Professor Kefeng Liu for his constant encouragement and valuable suggestions. 


\section{References}

[1] Q. M. Cheng and S. Ishikawa, A characterization of the Clifford torus, Proc. Amer. Math. Soc. 127 (1999), no. 3, 819-828.

[2] Q. M. Cheng and H. C. Yang, Chern's Conjecture on minimal hypersurfaces, Math. Z. 227 (1998), no. 3, 377-390.

[3] S. Y. Cheng and S.T.Yau, Hypersurfaces with constant scalar curvature, Math. Ann. 225 (1977), no. 3, 195-204.

[4] S. S. Chern, M. do Carmo, and S. Kobayashi, Minimal submanifolds of constant length, in Functional Analysis and Related Fields (F. E. Browder, ed.), Springer, New York (1970).

[5] B. Lawson, Local rigidity theorems for minimal hypersurfaces, Ann. of Math. (2) 89 (1969), 187-197.

[6] A. M. Li and J. M. Li, An intrinsic rigidity theorem for minimal submanifolds in a sphere, Arch. Math. (Basel) 58 (1992), no. 6, 582-594.

[7] K. Ogiue and H. F. Sun, Minimal hypersurfaces of unit sphere, Tohoku Math. J. 49 (1997), no. 3, 423-429.

[8] C. K. Peng and C. L. Terng, Minimal hypersurfaces of sphere with constant scalar curvature, Ann. of Math. Stud. 103 (1983), 177-198.

[9] _ The scalar curvature of minimal hypersurfaces in spheres, Math. Ann. 266 (1983), no. $1,105-113$.

[10] J. Simons, Minimal varieties in Riemannian manifolds, Ann. of Math.(2) 88 (1968), 62-105.

[11] $\mathrm{H} . \mathrm{W} . \mathrm{Xu}$, A rigidity theorem for submanifolds with parallel mean curvature in a sphere, Arch. Math. (Basel) 61 (1993), no. 5, 489-496.

[12] _ On closed minimal submanifolds in pinched Riemannian manifolds, Trans. Amer. Math. Soc. 347 (1995), no. 5, 1743-1751.

[13] H. W. Xu, W. Fang and F. Xiang, A generalization of Gauchman's rigidity theorem, Pacific J. Math. 228 (2006), no. 1, 185-199.

[14] S. T. Yau, Submanifolds with constant mean curvature, I, II, Amer. J. Math. 96 (1974), 346$366 ; 96$ (1975), 76-100.

Center of Mathematical Sciences, Zhejiang University, Hangzhou 310027, China

E-mail address: tobia_wsm@yahoo.com.cn

Center of Mathematical Sciences, Zhejiang University, Hangzhou 310027, China

E-mail address: xuhw@cms.zju.edu.cn 